\title{
Phosphorothioate cap analogs increase stability and translational efficiency of RNA vaccines in immature dendritic cells and induce superior immune responses in vivo
}

\author{
AN Kuhn ${ }^{1}$, M Diken ${ }^{1}$, S Kreiter ${ }^{1}$, A Selmi ${ }^{1}$, J Kowalska ${ }^{2}$, J Jemielity ${ }^{2}$, E Darzynkiewicz ${ }^{2}$, C Huber $^{1}$, \\ Ö Türeci ${ }^{1,3}$ and U Sahin ${ }^{1,3}$ \\ ${ }^{1}$ Division of Translational and Experimental Oncology, Department of Internal Medicine III, Johannes Gutenberg-University, Mainz, \\ Germany and ${ }^{2}$ Division of Biophysics, Faculty of Physics, Institute of Experimental Physics, University of Warsaw, Warsaw, Poland
}

\begin{abstract}
Vaccination with in vitro transcribed RNA coding for tumor antigens is considered a promising approach for cancer immunotherapy and has already entered human clinical testing. One of the basic objectives for development of RNA as a drug is the optimization of immunobioavailability of the encoded antigen in vivo. By analyzing the effect of different synthetic 5' mRNA cap analogs on the kinetics of the encoded protein, we found that $m_{2}^{7,2^{\prime}-O} G p p_{s} p G(\beta-S-A R C A)$ phosphorothioate caps, in particular the D1 diastereoisomer, profoundly enhance RNA stability and translational efficiency
\end{abstract}

Keywords: vaccination; dendritic cells; RNA; cap analog

\section{Introduction}

Nucleic acids with optimized properties are increasingly analyzed as a tool to deliver the genetic information of complete antigens for HLA haplotype-independent antigen-specific vaccination against cancer, autoimmune and infectious diseases. ${ }^{1,2}$ Compared with DNA, RNA has several advantages, for example, RNA cannot be integrated into the genome, and it gives rise to transient expression of the encoded proteins as desired for a pharmaceutical. Furthermore, recombinant RNA is easy to produce in large amounts and high purity by in vitro transcription, further enhancing its appeal as a drug compound. ${ }^{3}$

Combining this multifaceted antigen delivery vehicle with dendritic cells (DCs), the most potent antigenpresenting cells, is reckoned as a powerful approach to induce potentially therapeutic immune responses. ${ }^{4,5}$ Preclinical experiments and human clinical trials testing administration of autologous DCs loaded in vitro with RNA as well as direct injection of RNA via different

Correspondence: Professor U Sahin, Division of Translational and Experimental Oncology, Department of Internal Medicine III, Johannes Gutenberg-University, Obere Zahlbacher Str. 63, Mainz, 55131, Germany.

E-mail: sahin@uni-mainz.de

${ }^{3}$ These authors contributed equally to this work.

Received 20 December 2009; revised 28 February 2010; accepted 3 March 2010; published online 22 April 2010 in immature but not mature dendritic cells. Moreover, in vivo delivery of the antigen as $\beta-S-A R C A(D 1)$-capped $R N A$ species is superior for protein expression and for efficient priming and expansion of naive antigen-specific $T$ cells in mice. Our findings establish $5^{\prime}$ mRNA cap analogs as yet another module for tuning immunopharmacological properties of recombinant antigen-encoding $R N A$ for vaccination purposes.

Gene Therapy (2010) 17, 961-971; doi:10.1038/gt.2010.52; published online 22 April 2010

routes to reach DCs in situ showed feasibility, lack of toxicity and induction of the expected antigen-specific immune response. ${ }^{6-11}$

Improvement of immunobioavailability of RNA-based vaccines in DCs has been a recurrent subject of our research, because the dose of the antigen may be one of the critical factors for generating strong and sustained antigen-specific immune responses. ${ }^{12}$ In previous work, we have developed plasmid templates for in vitro transcription of RNA-encoded antigen with modified $3^{\prime}$ structures ( $3^{\prime}$ UTR and poly(A)tail) stabilizing the RNA and optimizing its translational performance. ${ }^{13}$ Moreover, we achieved significant leverage of major histocompatibility complex (MHC) class I and II presentation of the antigen in DCs by introducing routing signals. ${ }^{14} \mathrm{By}$ combining these measures, we were able to profoundly improve properties of RNA-encoded vaccines.

Yet another important structural element of in vitro transcribed RNA is the $5^{\prime}$ cap 7-methylguanosine $\left(\mathrm{m}^{7} \mathrm{G}\right)$ that is added to the first transcribed nucleotide via a $5^{\prime}$ to $5^{\prime}$ triphosphate bridge. ${ }^{15}$ This cap has important roles in all aspects of mRNA metabolism such as specific recognition of mature mRNA by the translational initiation factor eIF4E for formation of the 48S complex, translation initiation and stability of the RNA in the process of protein synthesis. ${ }^{16}$ Capping of recombinant antigen-encoding RNA manufactured for preclinical development and clinical use is achieved by transcribing the DNA template with a bacteriophage RNA 
polymerase in the presence of all four nucleotide triphosphates and the cap dinucleotide $\mathrm{m}^{7} \mathrm{GpppG}$ as structural homolog of the endogenous cap structure. ${ }^{3}$ However, not all of the RNA is capped, because the cap dinucleotide has to compete with guanosine triphosphate (GTP) for the initiation of transcription. In addition, $\mathrm{m}^{7} \mathrm{GpppG}$ is incorporated in both orientations into the RNA by the RNA polymerase, yielding $\mathrm{Gppm}^{7} \mathrm{GpN}$ as well as $\mathrm{m}^{7} \mathrm{GpppGpN}$ after addition of the first nucleotide. ${ }^{17}$ Therefore, more than half of the in vitro transcribed $\mathrm{m}^{7} \mathrm{GpppG}$-capped RNA molecules are not recognized by the translational machinery, resulting in decrease of the overall translational activity.

Several synthetic cap analogs have been described, which do not have the risk of reverse incorporation when co-transcriptionally attached, and do not compromise translation of the RNA. For example, 'two-headed' cap analogs of the format $\mathrm{m}^{7} \mathrm{Gp}_{(\mathrm{n})} \mathrm{m}^{7} \mathrm{G}$, owing to their symmetry, are exclusively incorporated in the correct orientation. ${ }^{18}$ Among such symmetric analogs the tetraphosphate $m^{7} \mathrm{Gppppm}^{7} \mathrm{G}$ was shown to have the highest translational efficiency in vitro. Alternatively, substitution of one of the $\mathrm{OH}$-groups in $\mathrm{C}^{\prime}$ or $\mathrm{C}^{\prime}$ position of the $\mathrm{m}^{7} \mathrm{G}$ with $\mathrm{OCH}_{3}$ overcomes reverse incorporation by completely blocking elongation at the methylated guanosine. ${ }^{19,20}$ The respective dinucleotides $\mathrm{m}_{2}^{7,2^{\prime}-\mathrm{O}} \mathrm{GpppG}$ and $\mathrm{m}_{2}^{7,3^{\prime}-\mathrm{O}} \mathrm{GpppG}$ are therefore called 'anti-reverse cap analogs' or ARCA. In addition to substitutions that block reverse incorporation, synthetic cap dinucleotides can be further modified to shape stability and translational characteristics of the respective RNA species. Substitution of a non-bridging oxygen in the $\beta$-phosphate moiety by sulfur results in the $\beta$-S-ARCA dinucleotide with its diastereoisomers named D1 and D2. RNAs capped with $\beta$-S-ARCA are less susceptible to degradation by human Dcp2, the nuclease that initiates the $5^{\prime}$ to $3^{\prime}$ decay of mRNAs, while maintaining recognition by the translational machinery. ${ }^{21,22}$

Up to now, such modified cap analogs have been primarily used to study RNA metabolism. Their effect, however, on bioavailability of the encoded protein in antigen-presenting cells such as DCs and eventually on induction of potent $\mathrm{T}$ cell responses in vivo has never been analyzed. The aim of this study was to preclinically evaluate novel cap analogs for immunopharmacological improvement of vaccines based on antigen-encoding RNA. We show that RNAs capped with the D1 diastereoisomer of $\beta$-S-ARCA have increased stability and translational efficiency in immature but not mature DCs. Accordingly, in vivo delivery of the antigen as $\beta$-SARCA(D1)-capped RNA species led to increased protein expression and enhanced priming and expansion of naïve antigen-specific T cells in mice. We expect that our findings pave the way for inauguration of modified cap analogs into RNA vaccine development and will likely contribute to a better immunization.

\section{Results}

\section{$\beta$-S-ARCA(D1)-capped RNA is superior in ensuring high and long-lasting protein expression in immature DCs}

To assess the effect of alternative $5^{\prime}$ RNA caps (Figure 1) on protein expression, equal amounts of differentially
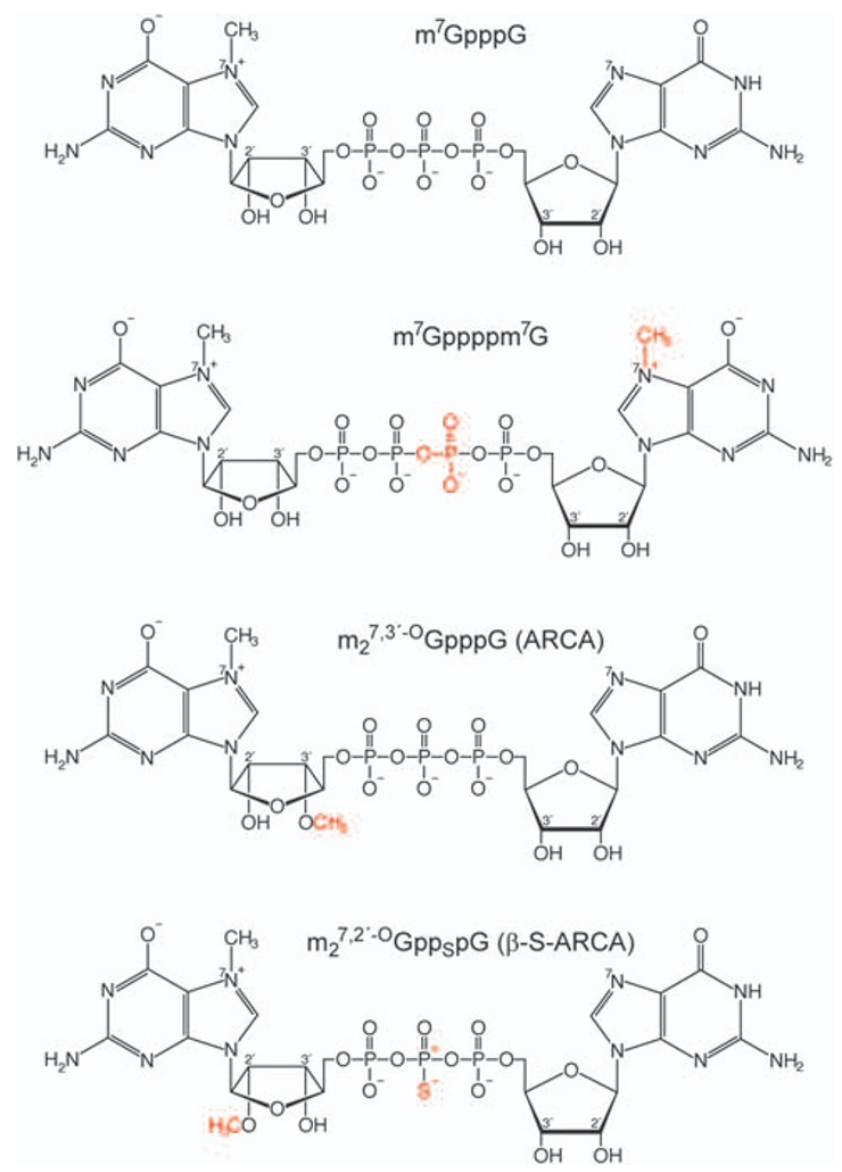

Figure 1 Functional cap analogs used in this study. Owing to the presence of a stereogenic $\mathrm{P}$ center (labeled with *), the phosphorothioate cap analog $\beta$-S-ARCA exists in two diastereomers. On the basis of their elution order in reverse phase high-performance liquid chromatography (HPLC), these have been designated as D1 and D2. ${ }^{22}$

capped RNA species encoding reporter proteins (luciferase or d2eGFP) and equipped with stabilizing $3^{\prime}$ ends ${ }^{13}$ were electroporated into human immature as well as mature DCs, which are considered as the in vitro cell system for testing the physiological activity of antigenencoding RNA vaccines. The activity of the reporter gene product was plotted as a function of time (Figure 2) to record decay kinetics of the respective protein. By mathematical modeling, parameters relevant for translational output such as translational efficiency (the maximal slope), relative functional mRNA stability (time point of the maximal protein expression) and total protein expression over time (integral of the curve) were deduced from individual curves (Table 1). Artificial cap analogs $\mathrm{m}^{7} \mathrm{Gppppm} \mathrm{m}^{7} \mathrm{G}, \mathrm{m}_{2}^{7,3^{\prime}-\mathrm{O}} \mathrm{GpppG}$ (ARCA), the D1 and D2 diastereoisomers of the phosphorothioate $\mathrm{m}_{2}^{7,2^{\prime}-O} \mathrm{Gpp}$ s pG ( $\beta$-S-ARCA), and $\mathrm{m}^{7} \mathrm{GpppG}$, the structural homolog of the natural cap, were linked co-transcriptionally to reporter RNA. As $\mathrm{m}^{7} \mathrm{GpppG}$ can be incorporated in both orientations, only approximately $50 \%$ of the capped RNAs in this specimen (named $\mathrm{m}^{7} \mathrm{GpppG}$ / c.t.) are translationally competent. For comparability, we included RNA-capped post-transcriptionally by the capping enzyme from vaccinia virus with $\mathrm{m}^{7} \mathrm{GpppG}$ (named $\mathrm{m}^{7} \mathrm{GpppG} / \mathrm{p} . \mathrm{t}$.). Both $\mathrm{m}^{7} \mathrm{GpppG}$-capped reporter RNA preparations irrespective of the technique used for attachment of the cap (c.t. and p.t.) showed 

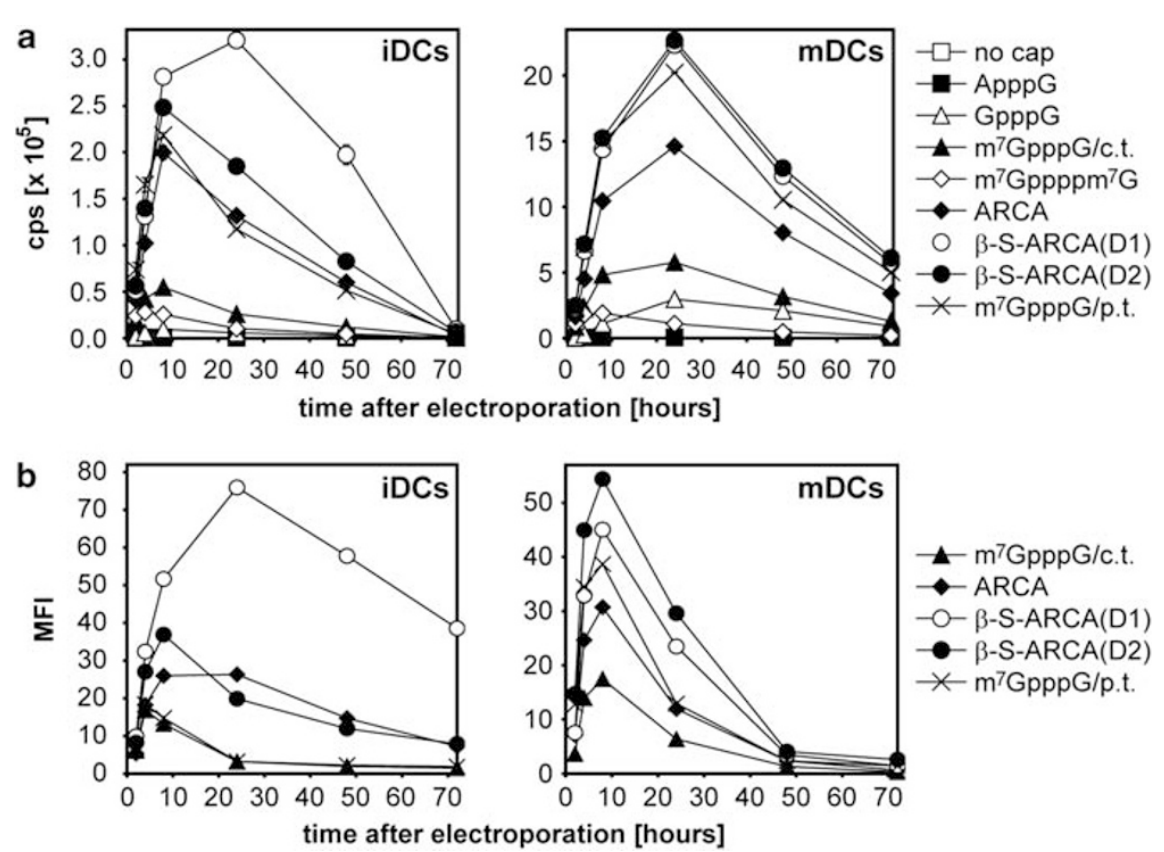

Figure 2 Effect of the $5^{\prime}$ mRNA cap structure on expression of the encoded protein in DCs. (a, b) Human immature and mature DCs (iDCs and $\mathrm{mDCs}$, respectively) were electroporated with equal amounts of RNAs encoding reporter genes (luciferase or d2eGFP) transcribed in the presence of the indicated cap analogs or capped post-transcriptionally using the capping enzyme from vaccinia virus ( $\mathrm{m}^{7} \mathrm{GpppG} / \mathrm{p} . \mathrm{t}$.). ApppG with adenosine instead of guanosine, and GpppG, which lacks the methyl-group essential for recruitment of the translational machinery, were used as non-functional cap controls. (a) Luciferase-encoding RNAs were used as reporters, luciferase activity (given in counts per second, cps) was measured, and the average values \pm s.d. are shown as a function of time. On the basis of the data shown here the translational efficiency, the time-point of the maximal protein expression, and the total protein expression as given in Table 1 were calculated. (b) d2eGFP-encoding RNAs were used as reporters, and fluorescence given in mean fluorescence intensity was measured by flow cytometry. For unknown reasons the expression from $\mathrm{m} 7 \mathrm{GpppG} / \mathrm{p}$.t. RNA was unusually low in immature DCs in the particular experiment shown here.

Table 1 Effect of the $5^{\prime}$ mRNA cap structure on translational properties

\begin{tabular}{|c|c|c|c|c|}
\hline $5^{\prime}$ Cap structure & Cells & Transl. efficiency & Time of max. (hours) & Total protein expression \\
\hline No cap & iDCs & $0.00 \pm 0.00$ & NA & $0.011 \pm 0.000$ \\
\hline ApppG & iDCs & $0.01 \pm 0.00$ & NA & $0.022 \pm 0.001$ \\
\hline GpppG & iDCs & $0.18 \pm 0.01$ & $11.5 \pm 0.0$ & $0.221 \pm 0.001$ \\
\hline $\mathrm{m}^{7} \mathrm{GpppG} /$ c.t. & iDCs & $1.00 \pm 0.04$ & $8.1 \pm 1.1$ & $1.000 \pm 0.007$ \\
\hline $\mathrm{m}^{7} \mathrm{Gppppm}^{7} \mathrm{G}$ & iDCs & $0.20 \pm 0.10$ & $4.9 \pm 0.1$ & $0.404 \pm 0.004$ \\
\hline ARCA & iDCs & $2.52 \pm 0.19$ & $12.6 \pm 0.1$ & $4.777 \pm 0.042$ \\
\hline$\beta$-S-ARCA(D1) & iDCs & $3.36 \pm 0.09$ & $15.4 \pm 0.1$ & $13.094 \pm 0.307$ \\
\hline$\beta-S-A R C A(D 2)$ & iDCs & $3.53 \pm 0.17$ & $12.8 \pm 0.0$ & $6.570 \pm 0.075$ \\
\hline $\mathrm{m}^{7} \mathrm{GpppG} /$ p.t. & iDCs & $4.12 \pm 0.53$ & $8.4 \pm 1.4$ & $4.289 \pm 0.056$ \\
\hline No cap & $\mathrm{mDCs}$ & $0.00 \pm 0.00$ & NA & $0.002 \pm 0.000$ \\
\hline ApppG & $\mathrm{mDCs}$ & $0.01 \pm 0.00$ & NA & $0.008 \pm 0.000$ \\
\hline GpppG & $\mathrm{mDCs}$ & $0.31 \pm 0,02$ & $27.5 \pm 0.5$ & $0.593 \pm 0.005$ \\
\hline $\mathrm{m}^{7} \mathrm{GpppG} /$ c.t. & $\mathrm{mDCs}$ & $1.00 \pm 0.05$ & $16.0 \pm 0.3$ & $1.000 \pm 0.003$ \\
\hline $\mathrm{m}^{7} \mathrm{Gppppm}^{7} \mathrm{G}$ & $\mathrm{mDCs}$ & $0.56 \pm 0.04$ & $6.0 \pm 0.3$ & $0.176 \pm 0.001$ \\
\hline ARCA & $\mathrm{mDCs}$ & $2.16 \pm 0.00$ & $17.5 \pm 0.3$ & $2.526 \pm 0.015$ \\
\hline$\beta$-S-ARCA(D1) & $\mathrm{mDCs}$ & $3.05 \pm 0.14$ & $20.1 \pm 0.1$ & $3.884 \pm 0.032$ \\
\hline$\beta-S-A R C A(D 2)$ & $\mathrm{mDCs}$ & $3.30 \pm 0.03$ & $19.4 \pm 0.1$ & $4.042 \pm 0.053$ \\
\hline $\mathrm{m}^{7} \mathrm{GpppG} /$ p.t. & $\mathrm{mDCs}$ & $3.16 \pm 0.08$ & $17.5 \pm 0.3$ & $3.421 \pm 0.010$ \\
\hline
\end{tabular}

Abbreviations: ARCA, anti-reverse cap analogs; DCs, dendritic cells; NA, not applicable.

Curves presented in Figure 2a were analyzed by mathematical modeling to determine translational efficiency (slope of the curve), time-point of maximal protein amount as indicator of functional RNA stability, and the total amount of protein (integral of the curve) in human immature and mature DCs (iDCs and mDCs, respectively). For each cell type, the translational efficiency and the total signal was set to 1 for the mRNA co-transcriptionally capped with $\mathrm{m}^{7} \mathrm{GpppG}$. Listed are average values \pm s.d.

comparable functional RNA stability in DCs, whereas they differed in their translational efficiency and therefore also the total protein yield (Table 1).
In human immature DCs, $\beta$-S-ARCA(D1)-capped luciferase RNA delivered highest and longest lasting activity of luciferase (Figure 2a and Table 1). Total protein 
amount produced from this RNA species was more than one order of magnitude higher as compared with $\mathrm{m}^{7} \mathrm{GpppG} / \mathrm{c.t}$. RNA and more than threefold of $\mathrm{m}^{7} \mathrm{GpppG} /$ p.t. RNA. Second best but clearly inferior was $\beta$-S-ARCA(D2)-capped RNA, which delivered half of the total protein amount obtained by its diastereoisomer. ARCA-capped RNA followed closely with protein yields nearly five times higher than with $\mathrm{m}^{7} \mathrm{GpppG}$ c.t., but only one third of D1-capped RNA. RNA species linked to phosphorothioate and ARCA cap variants appeared to profit from both a higher functional stability and higher translational efficiency (Table 1). Surprisingly, $\mathrm{m}^{7} \mathrm{Gppppm} \mathrm{m}^{7} \mathrm{G}$-capping of RNA, although reported to augment translation in vitro, ${ }^{18}$ resulted in very low protein yields, apparently because of impaired translation and instability of this RNA species in immature DCs.

In contrast to the significant improvement of protein yield achieved in human immature DCs by phosphorothioate capping, the effect of the various $5^{\prime}$ caps was less pronounced in human mature DCs (Figure 2a and Table 1). Interestingly, in these cells stability of RNA capped with the endogenous homolog $\mathrm{m}^{7} \mathrm{GpppG} / \mathrm{c}$.t. was more than two times higher than in immature DCs and not substantially augmented by ARCA-based caps, indicating that in mature DCs the translational efficiency is the major determinant for overall protein expression. Highest protein yield in mature DCs was achieved by $\beta$-S-ARCA(D2), which only slightly outperformed the D1 diastereoisomer, but had a clear benefit over the $\mathrm{m}^{7} \mathrm{GpppG} /$ c.t. cap (Table 1).

All findings were reassessed by protein decay kinetics using d2eGFP-encoding RNAs as measured by flow cytometry, and confirmed superiority of $\beta$-S-ARCA(D1) in human immature DCs and of the D2 diastereosiomer in human mature DCs (Figure 2b).

\section{$\beta$-S-ARCA(D1)-capped RNA has a longer physical half-life in immature $D C s$}

Our findings suggested that the superior protein yield from RNA species equipped with phosphorothioate cap analogs in human immature DCs is attributable to improvement of both translational efficiency as well as stability of the RNA. The latter was deduced from the time point, at which peak levels of protein were reached. This is a measure of functional rather than physical RNA stability, because it also depends on the translational competence of the RNA and the stability of the protein. As a more direct parameter for physical stability, we measured factual half-life of differentially capped RNAs. Human immature and mature DCs were electroporated with equal amounts of d2eGFP-encoding RNA species and the level of the foreign RNA was determined by realtime reverse transcriptase-PCR at different time points (Figure 3 and Table 2).

In human immature DCs, we observed biphasic degradation of all capped RNAs implying that the electroporation itself and/or presence of antigen-encoding in vitro transcribed RNA initiate cellular alterations, which affect RNA metabolism (Figure 3a and Table 2). This included ApppG-capped and thus translationally inactive RNA, ruling out involvement of the translation machinery in the degradation process. Within the first $8 \mathrm{~h}$ after electroporation, degradation was fast, but then switched into a much slower decay process. $\beta$-S-ARCA(D1)-capped RNA showed by far the longest half-life both in the early, as well as later phase with values of up to twofold of $\mathrm{m}^{7} \mathrm{GpppG} / \mathrm{c}$.t. RNA (Figure 3a and Table 2). Overall effects of the two other ARCA-based cap analogs on RNA stability were less pronounced. Interestingly, $\mathrm{m}^{7} \mathrm{Gppppm} \mathrm{m}^{7} \mathrm{G}$-capped RNA underwent dramatic degradation within the first few hours. The very small amount that was still present after $8 \mathrm{~h}$ was as stable as other capped RNAs, suggesting at least proper incorporation of this RNA species into ribosomes.

In human mature DCs, in contrast, we did not observe an initial phase of fast degradation and RNA decay kinetics were found to be monophasic. Accordingly, overall stability of RNA was higher. Interestingly, in mature DCs even un-capped RNA was found to be surprisingly stable with a half-life of more than $10 \mathrm{~h}$ as compared with $1.4 \mathrm{~h}$ in immature DCs. All tested functional cap analogs had modest effect on RNA stability in mature DCs. This confirmed our findings in reporter gene experiments (Figure 2 and Table 1), further supporting that in human mature DCs the major determinant for protein yield is the translational efficiency of the respective RNA.

\section{$\beta-S-A R C A(D 1)$-capped RNA is preferentially translated in immature $D C s$}

Having shown that physical stabilization of the RNA by a $\beta$-S-ARCA(D1) cap contributes to the superiority of such RNA in delivering high protein amounts, we next wanted to characterize the efficiency with which RNA species capped with this analog are recognized by the translation machinery in the cell. This can be best analyzed when translation factors become rate limiting. Such conditions can be achieved by introducing high amounts either of the RNA species under investigation or of a competitor RNA into the cell. To simulate the first case, we compared protein decay kinetics in human immature DCs transfected either with 40 pmol or 20 pmol of luciferase-encoding RNA capped with either ARCA or $\beta$-S-ARCA(D1) by calculating ratios of luciferase activities for each time point (Figure $4 \mathrm{a}$ ). Interestingly, for $\beta$-S-ARCA(D1)-capped RNA the level of luciferase protein was proportional to the amount of RNA that was used for electroporation over the whole $72 \mathrm{~h}$ of the experiment. The signal obtained from cells with 40 pmol RNA was approximately twice as high as the signal from those with 20 pmol RNA for each timepoint. In contrast, when ARCA-capped RNA was used, the luciferase activity measured in cells elecroporated with 40 pmol decreased relatively faster as compared with the signal from those with 20 pmol RNA with the ratio reaching to 1.2 -fold $72 \mathrm{~h}$ after electroporation (Figure 4a).

Similarly, co-electroporation of the same amount of d2eGFP-encoding RNA as competitor capped with either ARCA or $\beta$-S-ARCA(D1) into human immature DCs resulted in a significant drop in the expression of ARCA-capped but not $\beta$-S-ARCA(D1)-capped luciferaseencoding RNA as compared with immature DCs, which lacked such a competing d2eGFP RNA (Figure 4b). Taken together, these findings indicate that $\beta$-S-ARCA(D1)-capped RNA is much more efficient than 

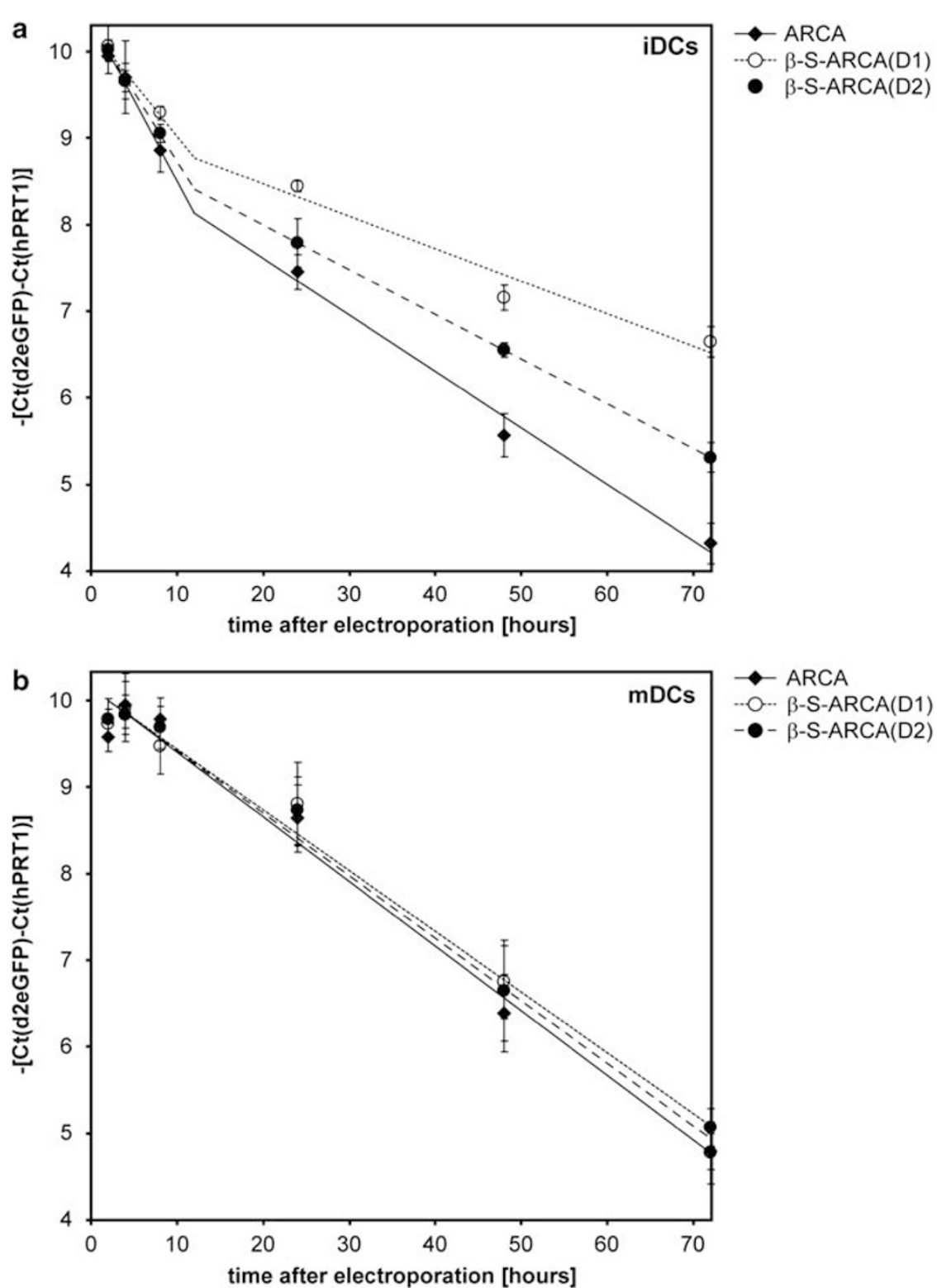

Figure 3 Effect of the $5^{\prime}$ cap on half-life and stability of mRNAs in DCs. (a) Human immature and (b) mature DCs (iDCs and mDCs, respectively) were electroporated with equal amounts of d2eGFP-encoding mRNAs capped with different cap analogs. Cells were harvested after 2, 4, 8, 24, 48 and $72 \mathrm{~h}$, RNA isolated from the cells, and the d2eGFP-transcript levels were quantified by real-time reverse transcriptasePCR (RT-PCR). For each time point, the difference between the threshold cycles (Ct) of RNAs encoding d2eGFP and hypoxanthine phosphoribosyltransferase (HPRT1) used as internal control was calculated. The data were fitted to a biphasic (immature DCs), or to a monophasic decay curve (mature DCs). The mRNA half-lifes calculated from the data shown here are listed in Table 2.

Table 2 Stability of differentially capped RNAs in human immature and mature DCs (iDCs and mDCs, respectively)

\begin{tabular}{|c|c|c|c|c|c|}
\hline $5^{\prime}$ Cap structure & Cells & $\begin{array}{c}m R N A \text { half-life ( } h) \\
\text { (2-8 h after electroporation) }\end{array}$ & $\begin{array}{l}m R N A \text { half-life }(h) \\
\text { (24-72 } h \text { after electroporation) }\end{array}$ & Cells & $\begin{array}{c}m R N A \text { half-life }(h) \\
\text { (2-72 } h \text { after electroporation) }\end{array}$ \\
\hline No cap & iDCs & $1.41 \pm 0.02$ & NA & $\mathrm{mDCs}$ & $10.36 \pm 0.18$ \\
\hline ApppG & iDCs & $5.98 \pm 0.49$ & $14.06 \pm 1.43$ & $\mathrm{mDCs}$ & $15.30 \pm 0.64$ \\
\hline GpppG & iDCs & $4.82 \pm 0.71$ & $24.45 \pm 4.40$ & $\mathrm{mDCs}$ & $14.64 \pm 0.32$ \\
\hline $\mathrm{m}^{7} \mathrm{GpppG} / \mathrm{c}$.t. & iDCs & $5.82 \pm 1.46$ & $16.10 \pm 1.83$ & $\mathrm{mDCs}$ & $13.11 \pm 0.82$ \\
\hline $\mathrm{m}^{7} \mathrm{Gppppm}^{7} \mathrm{G}$ & iDCs & $2.37 \pm 0.09$ & $19.37 \pm 0.34$ & mDCs & $11.88 \pm 0.39$ \\
\hline ARCA & iDCs & $5.47 \pm 0.87$ & $15.50 \pm 1.57$ & mDCs & $13.63 \pm 0.55$ \\
\hline$\beta$-S-ARCA(D1) & iDCs & $8.27 \pm 1.15$ & $27.00 \pm 2.85$ & mDCs & $13.94 \pm 0.82$ \\
\hline$\beta$-S-ARCA(D2) & iDCs & $6.72 \pm 1.48$ & $18.09 \pm 0.81$ & mDCs & $14.20 \pm 0.89$ \\
\hline $\mathrm{m}^{7} \mathrm{GpppG} / \mathrm{p} . \mathrm{t}$ & iDCs & $6.11 \pm 0.33$ & $15.60 \pm 7.24$ & mDCs & $13.27 \pm 0.28$ \\
\hline
\end{tabular}

Abbreviations: ARCA, anti-reverse cap analogs; DCs, dendritic cells; NA, not applicable.

Listed are average values \pm s.d. 


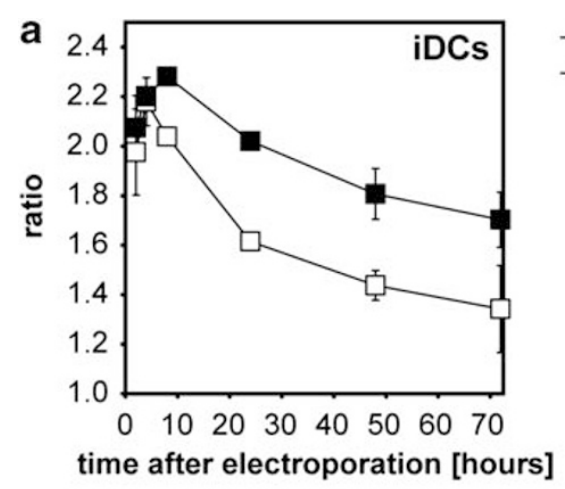

\author{
$-\square-40$ pmol ARCA-luc./20 pmol ARCA-luc. \\ - $-40 \mathrm{pmol} \beta-S-A R C A(D 1)-l u c . / 20 \mathrm{pmol}$ \\ $\beta$-S-ARCA(D1)-luc.
}
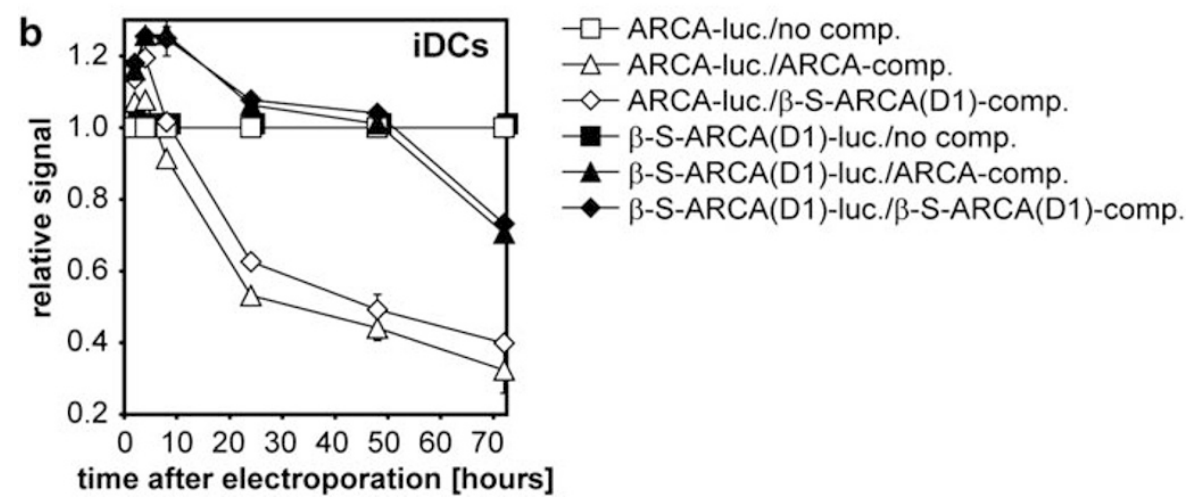

Figure 4 Competition for translation between ARCA- and $\beta$-S-ARCA(D1)-capped RNAs. Protein decay kinetics in human immature DCs were determined by measuring luciferase activities at different time points after electroporation with the indicated RNA species. (a) Ratios of luciferase activities measured at the respective time points in cells electroporated with either 40 or 20 pmol of RNAs equipped with the same cap ( \pm s.d. of two independent experiments). (b) Cells were electroporated with luciferase RNA alone or together with d2eGFP RNA as competitor (comp.) each equipped with the indicated cap. Luciferase activity was recorded and set in relation to luciferase activity of cells electroporated exclusively with the respective luciferase species without competitor to obtain the 'relative signal' (set to 1 for both ARCA- and $\beta$-S-ARCA(D1)-capped RNA).

ARCA-capped RNA to compete with endogenous RNA for the translational machinery and thus is preferentially translated in human immature DCs.

\section{$\beta-S-A R C A(D 1)$-capped RNA gives rise to increased protein expression in vivo}

Our in vitro findings so far showed that in human immature DCs $\beta$-S-ARCA(D1)-capped RNA profits from longer RNA half-life and higher translational efficiency and thus provides significantly higher amounts of the encoded protein for a prolonged time. Next, we wanted to know whether these mechanisms were relevant when naked RNA is in vivo administered. For this purpose, luciferase-encoding RNA capped either with ARCA or $\beta$-S-ARCA(D1) was injected into the inguinal lymph node of mice, and protein expression was quantified at different time points after RNA injection using in vivo bioluminescence imaging (Figure 5).

Consistent with our observations in vitro, we found significantly higher and sustained protein expression in mice injected with $\beta$-S-ARCA(D1)-capped RNA, with total protein expression being eightfold higher as compared with ARCA-capped RNA. Robust levels of protein were already detected within the first $2 \mathrm{~h}$ and persisted 2 days (Figure 5a). As the curves of the average values (Figure $5 \mathrm{~b}$ ) both peak at the same time point, it appears as if higher protein amounts of $\beta$-S-ARCA(D1)capped RNA are mediated primarily by an increase in translation efficiency. However, in three of the nine mice treated with $\beta$-S-ARCA(D1)-capped RNA, the maximal amount of luciferase was measured at later time-points of up to more than $19 \mathrm{~h}$ after RNA administration (Figure 5c). In mice with ARCA-capped RNA, we never observed luciferase peaks later than $12.5 \mathrm{~h}$, suggesting that $\beta$-S-ARCA(D1) has at least partly a contribution to higher RNA stability.

Immunization with $\beta$-S-ARCA(D1)-capped RNA results in efficient de novo priming of antigen-specific $T$ cells Next, we analyzed the effect of enhanced antigen delivery by $\beta$-S-ARCA(D1)-capped RNA on the induction of antigen-specific immune responses in vivo. We resorted to a model system, which we have recently used to measure de novo priming of naïve $\mathrm{T}$ cells on immunization with RNA-loaded DCs. ${ }^{14}$ RNA encoding the SIINFEKL epitope of chicken ovalbumin, inserted in between secretory and transmebrane domains of MHC class I (sec-SIINFEKL-MITD) for optimized presentation on antigen-presenting cells, was capped with $\beta$-SARCA(D1) or ARCA cap analogs. Mice were immunized twice (day 0 and day 3) by intranodal injection of sec-SIINFEKL-MITD RNA species.

In peripheral blood as well as in spleen, the percentage of CD8 ${ }^{+} \mathrm{T}$ cells as measured by MHC-tetramer staining on day 8 was up to threefold higher in mice immunized with $\beta$-S-ARCA(D1)-capped RNA as compared with the 


\section{a}
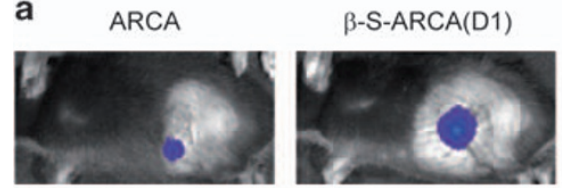

time after injection

2 hours
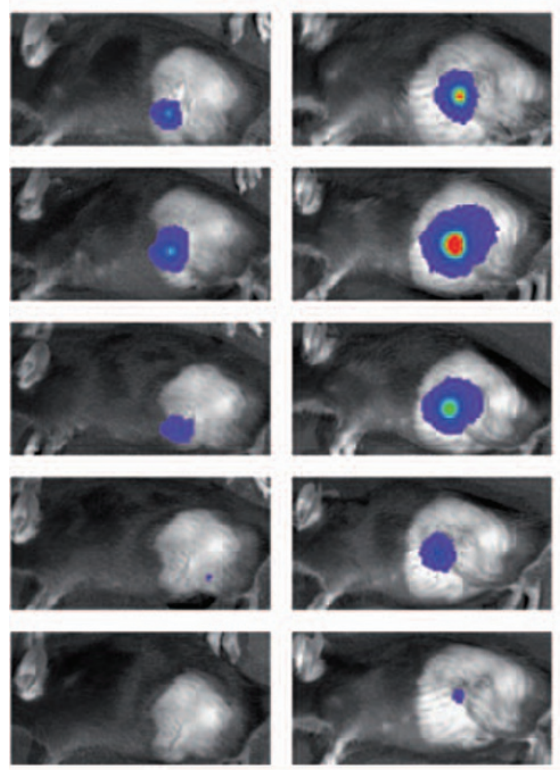

pps $\left[\times 10^{6}\right.$
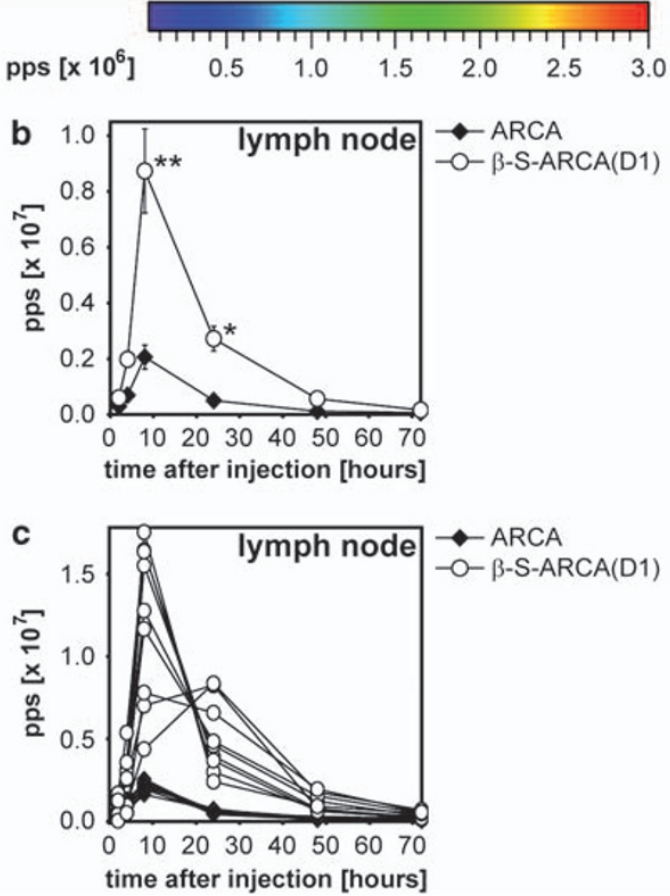

Figure 5 Effect of the $5^{\prime}$ cap on protein expression in vivo. Two groups of mice (each $n=9$ ) received injections of equal amounts of luciferase-encoding mRNA with either ARCA or $\beta$-S-ARCA(D1) $5^{\prime}$ cap structures into inguinal lymph nodes. In vivo imaging of luciferase activity (given in pps) was performed at different time points. (a) Imaging series of representative mice. The photon counts are represented as given by the color scale at the bottom. (b) Time kinetics of average luciferase activity. Error bars indicate standard error of the mean (s.e.m.). Statistical analysis (Student's $t$-test) was performed for the measurements after 8 and $24 \mathrm{~h}\left({ }^{*} P<0.005\right.$ and ${ }_{* *} P<0.02$ ). (c) Time kinetics of luciferase activities for all mice. For each cap analog, the curves were normalized based on the total protein expression to better visualize the functional RNA stability. a

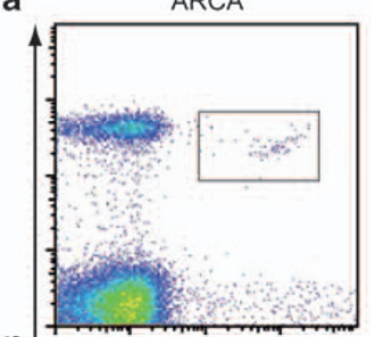

ơ
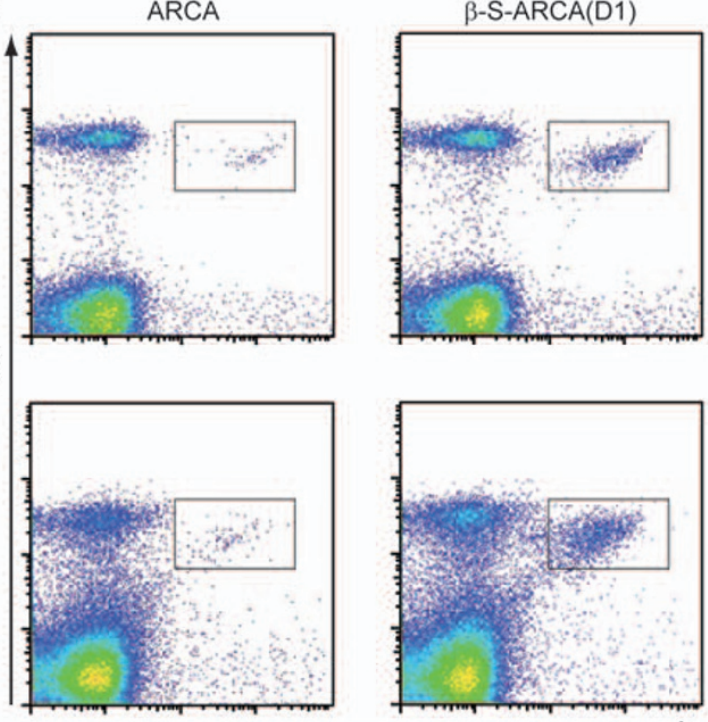

blood

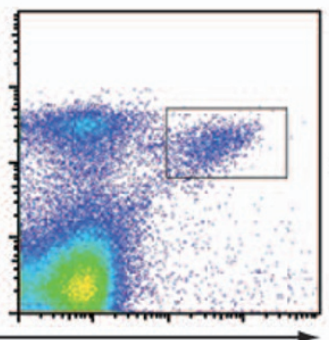

tetramer

b

ARCA

$\beta-S-A R C A(D 1)$

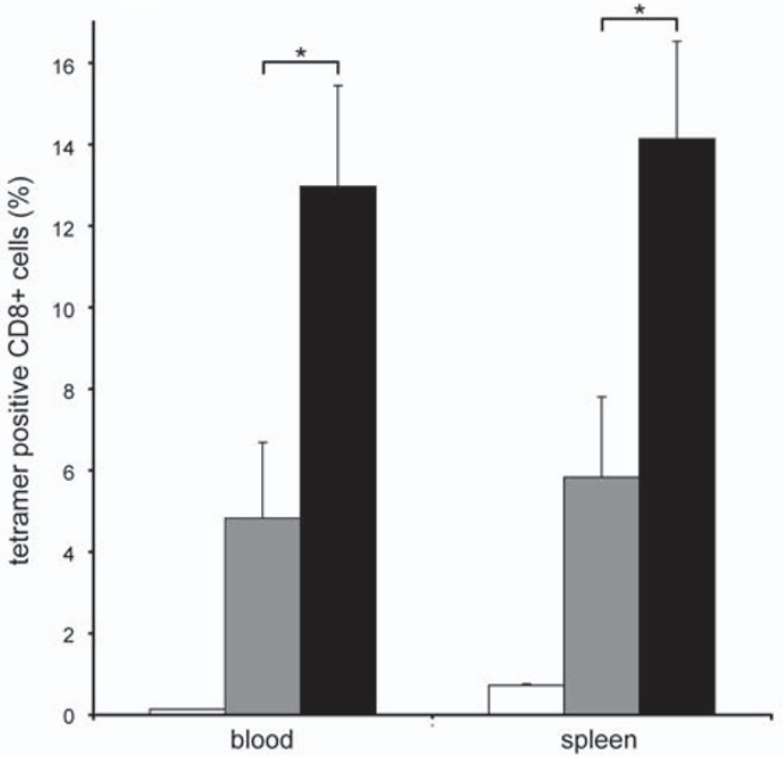

Figure 6 Effect of the 5' mRNA cap structure on de novo priming of naïve $T$ cells after intranodal immunization with antigen-encoding mRNA. Two groups of mice (each $n=5$ ) were intranodally immunized twice (day 0 and day 3 ) with equal amounts of SIINFEKL-encoding mRNA capped either with ARCA or $\beta$-SARCA(D1). The frequency of SIINFEKL-specific CD8+ T cells was quantified by tetramer staining on day 8. As a control, one group of mice $(n=3)$ was treated with buffer (no RNA) (a) Representative dot-plots of cells from peripheral blood and spleen of immunized mice are shown. (b) Average number of tetramer positive CD8+ cells (in \%) as measured on day 8 . Error bars indicate standard error of the mean (s.e.m.). Statistical analysis (Student's $t$-test) was performed $\left({ }^{*} P<0.075\right)$.

control group receiving ARCA-capped RNA (Figures 6a, b). Most interestingly, more than 12.5 and $14.0 \%$ of the CD8+ T cells in blood and spleen, respectively, were found to be antigen-specific. In summary, our data underline that the use of $\beta$-S-ARCA(D1) for 
capping RNA ensures highly efficient priming and profound expansion of naïve $\mathrm{T}$ cells from a natural repertoire.

\section{Discussion}

The use of antigen-encoding RNA provides the opportunity to address several of the shortcomings of current vaccination strategies at once. The success and the ultimate clinical usefulness of this approach, however, will depend on the optimization of parameters contributing to induction and efficient expansion of $\mathrm{T}$ cell responses. Our aim in this study was to refine the stability and translational performance of transiently expressed RNA in DCs and thus to improve the pharmacokinetics of antigen delivery. This was achieved by evaluating the effect of modified $5^{\prime}$ mRNA cap structures on the pharmacology of antigen-encoding RNA as active ingredient of an RNA vaccine.

We selected $\mathrm{m}^{7} \mathrm{Gppppm} \mathrm{m}^{7} \mathrm{G}$, ARCA and the two diasteroisomers of $\beta$-S-ARCA for two reasons. First, in contrast to $\mathrm{m}^{7} \mathrm{GpppG}$, the current gold-standard for clinical applications of RNA, these cap analogs were designed to be incorporated solely in the correct orientation during in vitro transcription by the bacteriophage RNA polymerase, thereby increasing the portion of translationally competent in vitro transcribed RNAs. ${ }^{18-20}$ Second, these cap analogs contain modifications that have been shown to increase translational efficiency and stability when present at the $5^{\prime}$ end of mRNAs. ${ }^{21,22}$ However, up to now these cap analogs had been primarily studied in cell-free systems for their biochemical characteristics, thus there is scarcity of information regarding their performance in cells. A putative benefit for RNA-based vaccines by modified cap analogs was suggested in a review that was published while this work was in progress. ${ }^{2}$ Only two reports have been published, in which the effect of ARCA on protein expression in DCs was analyzed in comparison with $\mathrm{m}^{7} \mathrm{GpppG} .^{23,24}$ By analyzing the effect of the different cap analogs on the half-life and translational efficiency of RNAs in human immature and mature DCs, as well as antigen-specific T cell response on vaccination with RNA in mice to enable read-outs as close as possible to the desired clinical effects, we made several surprising findings.

First, we showed that among the set of tested cap analogs, $\beta$-S-ARCA(D1) is significantly superior in ensuring expression of the encoded antigen in immature DCs in vitro and in vivo. This was surprising, because in the only previous report that compared translational performance of RNA species capped with the three ARCA-derived analogs in HC11 cells, RNA capped with the D2 diastereoisomer had been described as superior. ${ }^{21}$ $\beta$-S-ARCA(D1) and (D2) differ in two features, namely that the D1 diastereoisomer has a higher affinity to the cap-binding translation initiation factor eIF4E, ${ }^{22}$ while RNA capped with $\beta$-S-ARCA(D2) has been shown to be less susceptible to human Dcp2, the key component of the enzyme complex responsible for $5^{\prime}$ to $3^{\prime}$ decay of mRNAs. ${ }^{21}$ It is conceivable that depending on the cell system in which the capped RNAs are assessed, contributions of such features to the overall performance of the RNA do differ. According to our data, in vitro transcribed RNA profits particularly from better recruitment of the translation machinery in immature DCs, and less from a higher physical stability, because RNA capped with $\beta$-S-ARCA(D1) gives highest reporter gene expression. The stabilization of $\beta$-S-ARCA(D1)-capped RNA would thus be explained by efficient binding of eIF4E to the $5^{\prime}$ end, as previously shown. ${ }^{25}$ In contrast, in mature DCs the influence of RNA stabilization and affinity for eIF4E seems to be the opposite, because $\beta$-SARCA(D2)-capped RNA gives highest protein synthesis. In addition, our data can be observed as an indication for domination of $5^{\prime} \rightarrow 3^{\prime}$ rather than $3^{\prime} \rightarrow 5^{\prime}$ degradation routes in immature DCs. This assumption is based on the finding that protection of the $5^{\prime}$ end of an RNA against cleavage by Dcp 2 shifts the balance of $5^{\prime} \rightarrow 3^{\prime}$ and $3^{\prime} \rightarrow 5^{\prime}$ degradation to the latter. ${ }^{25}$

In a recent report that is consistent with our data, it was shown that ectopic CD40L expression in immature DCs is higher from post-transcriptionally capped RNA compared with ARCA-capped RNA. ${ }^{26}$ However, $\beta-S-$ ARCA(D1) (or D2) was not tested by these investigators, and thus the superiority of $\beta$-S-ARCA(D1)-capped RNAs over post-transcriptionally capped RNA on protein expression and RNA stability in immature DCs remained unrevealed.

Second, we observed that the range in which protein yield from RNA can be modulated by its $5^{\prime}$ cap depends on the cell type. This range appears to be broader in immature DCs compared with mature DCs. Moreover, our results show clearly that RNAs are in general more stable in mature DCs as compared with immature DCs. The most likely explanation is that both cell types differ considerably with regard to the complex network of RNA-protein interactions. In fact, as already discussed in a previous study from our laboratory, ${ }^{13}$ revisiting our database of genome-wide expression profiles of immature and mature human DCs obtained with microarrays $^{27}$ has shown that factors involved in RNA metabolism are differentially regulated in the course of maturation of DCs.

Third, we have shown that optimization of translational performance of RNA by $\beta$-S-ARCA(D1) capping improves the capability of such an antigen-encoding RNA to induce immune responses and results in a three- to fourfold higher frequency of antigen-specific $\mathrm{T}$ cells in vivo after intranodal RNA immunization. Along with others, we have shown earlier that an increase in protein expression augments the density of peptide-MHC complexes presented on the cell surface, which finally results in superior T cell expansion. ${ }^{12,13}$ As both stability and translational efficiency are improved by $\beta$-S-ARCA(D1) capping, one would expect that an increase in both steady-state protein levels and defective ribosomal products defined as unfinished or misfolded proteins are relevant factors for the observed improvements. ${ }^{28}$

Fourth, a general lesson learned was the importance of testing cap analogs in a cell system suitable for the desired physiological activity. For example, we revealed that in human DCs yield from $\mathrm{m}^{7} \mathrm{Gppppm} \mathrm{m}^{7} \mathrm{G}$-capped RNA cells is even lower than from RNA co-transcriptionally capped with $\mathrm{m}^{7} \mathrm{GpppG}$. This is in stark contrast to results obtained using an in vitro translation system, in which this cap analogs augments translation compared with $\mathrm{m}^{7} \mathrm{GpppG}{ }^{18}$ This implies that either the 
methyl-group at the initiating guanosine or the tetraphosphate bridge or both seem to be inhibitory for recruitment of the translation apparatus in DCs.

The results of this study have important implications for the therapeutic use of antigen-encoding RNA. It has been shown previously in a diligently conducted study that DCs electroporated after maturation are more efficient in in vitro migration assays and at least as effective in antigen presentation as DCs transfected before maturation. ${ }^{29}$ On the basis of this notion and the kinetics of migration of mature DCs, which need 24 to $48 \mathrm{~h}$ to reach the lymph node, one would suggest to first mature and subsequently electroporate DCs for subcutaneous or intradermal injection. For this type of application both diastereoisomers D1 and D2 are of interest, because they profoundly increase bioavailiability of the antigen in mature DCs and ensure that antigen doses are still high when transfected DCs reach the side of $\mathrm{T}$ cell priming. The reason, however, why we are excited regarding the fact that in immature DCs $\beta$-S-ARCA(D1) cap analog profoundly enhances RNA stability and translational efficiency, is our particular interest in using the RNA naked and for direct injection, for example, intradermally or into the lymph node for uptake by local DCs. ${ }^{7-9}$

In summary, our data reveal $5^{\prime}$ mRNA cap analogs as a further structural feature to modify the stability and translational efficiency of in vitro transcribed RNA for pharmaceutical applications. Together with other modifications as previously described, ${ }^{13,14}$ we now have a tool-box in hand to design RNAs with properties suited for a diversity of applications, further enhancing the potential of RNA as a promising drug compound.

\section{Materials and methods}

\section{Animals}

C57BL/6 mice were purchased from Jackson Laboratories (Bar Harbor, ME, USA). All animal experiments complied with the guidelines set by the Johannes Gutenberg-University of Mainz.

\section{Cells}

Human immature DCs were differentiated from purified $\mathrm{CD}^{+} 4^{+}$monocytes of healthy donors in cytokine-supplemented culture as described previously. ${ }^{30}$ Maturation of human DCs was induced by culturing for 2 days with $500 \mathrm{U} \mathrm{ml}^{-1}$ IL-4 (Miltenyi Biotec, Bergisch Gladbach, Germany), $800 \mathrm{U} \mathrm{ml}^{-1}$ GM-CSF (Essex, Luzern, Switzerland), $10 \mathrm{ng} \mathrm{ml}^{-1}$ IL-1 $\beta$ (BD Biosciences, Heidelberg, Germany), $10 \mathrm{ng} \mathrm{ml}^{-1}$ TNF- $\alpha$ (Sigma, Taufkirchen, Germany), $1000 \mathrm{U} \mathrm{ml}^{-1}$ IL-6 (Miltenyi Biotec) and $1 \mu \mathrm{g} \mathrm{ml}^{-1}$ PGE2 (Sigma).

\section{Vectors for in vitro transcription}

Plasmid constructs used as templates for in vitro transcription of RNAs encoding luciferase and d2eGFP were based on pST1-2hBgUTR-A120. ${ }^{13}$ Owing to a $3^{\prime}$ $\beta$-globin UTR and a poly (A) tail of 120 nucleotides, this vector allows generation of pharmacologically improved in vitro transcribed RNA. The pST1-sec-SIINFEKLMITD-2hBgUTR-A120 vector construct was described previously. ${ }^{14}$ It encodes for a protein tagged with a secretion signal (sec) and the transmembrane and cytosolic domains of the MHC class I (MITD) ensuring efficient presentation on MHC class I as well as class II molecules.

\section{Generation of in vitro transcribed RNA}

To generate templates for in vitro transcription, plasmids were linearized downstream of the poly(A) tail using a class IIs restriction endonuclease, thereby generating a template to transcribe RNAs with no additional nucleotides past the poly(A)-tail. ${ }^{13}$ Linearized vector DNAs were purified by phenol-chloroform extraction and ethanol precipitation, quantified spectrophotometrically, and subjected to in vitro transcription with T7 RNA polymerase using the MEGAscript T7 Kit (Ambion, Austin, TX, USA). For reactions with a cap analog, the GTP concentration was lowered to $1.5 \mathrm{mM}$, and $6 \mathrm{mM}$ of $\mathrm{m}^{7} \mathrm{GpppG}, \mathrm{m}^{7} \mathrm{G}$ ppppm ${ }^{7} \mathrm{G}$, ARCA, $\beta$-S-ARCA(D1), or $\beta$ S-ARCA(D2), synthesized as described previously, ${ }^{18,20,22}$ were added to the reaction. For post-transcriptional capping with the vaccinia virus capping enzyme, the ScriptCap $\mathrm{m}^{7} \mathrm{G}$ capping system (Epicentre Technologies, Madison, WI, USA) was used. RNA was purified using the MEGAclear kit according to the manufacturer's instructions (Ambion). RNA concentration and quality were assessed by spectrophotometry and analysis on a 2100 Bioanalyzer (Agilent, Santa Clara, CA, USA).

\section{Transfer of in vitro transcribed RNA into cells}

In all, $1-10 \times 10^{6}$ cells were suspended in $250 \mu \mathrm{l}$ X-VIVO 15 medium (Cambrex, Verviers, Belgium) and transferred to a 4-mm gap sterile electroporation cuvette (Bio-Rad, Hercules, CA, USA). The appropriate amount of in vitro transcribed RNA was added and cells were electroporated using a Gene-Pulser-II apparatus (Bio-Rad, Munich, Germany) with voltage and capacitance settings of $300 \mathrm{~V}$ per $150 \mu \mathrm{F}$. Cells were diluted in culture medium immediately after electroporation.

\section{Reporter gene assays}

For luciferase assays, human DCs electroporated with in vitro transcribed RNA coding for luciferase were plated in 96-well white microplates (Nunc, Langenselbold, Germany) and incubated at $37^{\circ} \mathrm{C}$. Lysis of cells was performed using the 'Bright-Glo Luciferase Assay System' (Promega, Madison, WI, USA). Bioluminescence flux was measured with the Infinite M200 microplate luminescence reader (Tecan, Crailsheim, Germany) with 1-s integration time. Data were represented in counts per second.

For quantification of d2eGFP protein, cells were washed in phosphate-buffered saline and incubated with propidium iodide $\left(10 \mu \mathrm{g} \mathrm{ml}^{-1}\right)$ before flow cytometric analysis on a FACSCalibur analytical flow cytometer (BD Biosciences). Gating was performed on cells showing large forward scatter and side scatter for exclusion of contaminating cells. Propidium iodide-negative (live) cells were gated, and mean fluorescence intensity expression was determined using CellquestPro software (BD Biosciences).

Both for luciferase and d2eGFP, the activity of the reporter gene product was plotted as a function of time. To calculate parameters for translational efficiency (maximal slope), functional RNA stability (time of maximal protein expression) and total protein expression (integral of the curve), data points were fitted by 
piecewise polynomial interpolation (also called spline interpolation), and the relevant parameters were deduced with R. ${ }^{31}$ Briefly, values are read into the program, and means and s.d. are calculated using the methods 'mean' and 's.d.', respectively. Then, spline objects are calculated from the mean values based on an interpolation spline using the method 'interpSpline', and from these the function itself is calculated with the method 'polySpline'. Both 'interSpline' and 'polySpline' are part of the R-package 'library('splines')'. Next, data points with a step of $0.25 \mathrm{~h}$ are estimated using 'predict', and the maximum (indicative for the functional RNA stability) is determined. Similarly, the first derivative, whose maximum determines the maximal slope of the original curve (indicative for the translational efficiency), is calculated. Finally, upper sum and lower sum of the function are calculated. The integral is then given by the average of these values. A detailed script is available on request.

\section{Quantification of d2eGFP-encoding RNA levels by real-time reverse transcriptase- $P C R$}

RNA extraction, first-strand complementary DNA synthesis, and real-time reverse transcriptase-PCR were performed essentially as described previously. ${ }^{32,33}$ Total cellular RNA was extracted from DCs with RNeasy Mini Kit (Qiagen, Hilden, Germany), reverse transcribed with oligo- $\mathrm{dT}_{18}$ using Superscript II (Invitrogen, Carlsbad, CA, USA), and subjected to real-time quantitative analysis on ABI PRISM 7700 Sequence Detection System instrument and software (Applied Biosystems, Foster City, CA, USA) with QuantiTect SYBR Green PCR Kit (Qiagen). Reactions were performed in duplicates with specific primers amplifying the d2eGFP-encoding region (eGFP-sense, 5'-CACATGAAGCAGCACGACTTC-3'; eGFPantisense, 5'-CACCTTGATGCCGTTCTTCTG-3'; each $300 \mathrm{nM}$ ) with initial denaturation/activation for $15 \mathrm{~min}$ at $95{ }^{\circ} \mathrm{C}$, and 40 cycles of $30 \mathrm{~s}$ at $94{ }^{\circ} \mathrm{C}, 30 \mathrm{~s}$ at $62^{\circ} \mathrm{C}$ and $30 \mathrm{~s}$ at $72{ }^{\circ} \mathrm{C}$. Expression of d2eGFP transcripts was quantified using $\Delta \mathrm{Ct}$ calculation relative to hPRT1encoding RNA as internal standard (hPRT1-sense, $5^{\prime}-\mathrm{TG}$ ACACTGGCAAAACAATGCA-3'; hPRT1-antisense, 5' -G GTCCTTTTCACCAGCAAGCT-3'; with PCR conditions identical as above) to normalize for variances in the quality of RNA and the amount of input complementary DNA.

\section{Intranodal injection of in vitro transcribed RNA into mice}

For intranodal immunization, mice were anaesthetized with xylazine-ketamine and the inguinal lymph node was surgically exposed. After injection of in vitro transcribed RNA in a volume of 5-10 $\mu \mathrm{l}$, the wound was closed.

\section{In vivo bioluminescence imaging}

Evaluation of uptake and translation of luciferaseencoding RNA was performed by in vivo bioluminescence imaging. Mice received intraperitoneally an aqueous solution of D-luciferin $\left(150 \mathrm{mg} \mathrm{kg}^{-1}\right.$ body weight) after being anesthetized with isofluorane. Photons emitted were collected for $1 \mathrm{~min}$ using an IVIS Lumina imaging system (Caliper Life Sciences, Alameda, CA, USA). Measured bioluminesce signal in regions of interest was quantified and presented as color-scaled images superimposed on grayscale photos of mice by using the Living Image software (Caliper Life Sciences). For absolute quantification, the bioluminescence signal retrieved from the lymph node was normalized by subtraction background luminescence signal from a non-signal emitting region.

\section{De novo priming of $T$ cells}

To determine the effect of $5^{\prime}$ mRNA cap analogs on de novo priming of T cells upon intranodal immunization with RNA, RNA encoding the SIINFEKL epitope of chicken ovalbumin, inserted in between secretory and transmebrane domains of MHC class I (sec-SIINFEKLMITD) for optimized presentation on antigen-presenting cells, ${ }^{14}$ was capped with ARCA or $\beta$-S-ARCA(D1) dinucleotides. After two intranodal immunizations of the respective sec-SIINFEKL-MITD RNA species on day 0 and day 3 into the same lymph node of mice, SIINFEKL-specific $\mathrm{T}$ cells were quantified on day 8 in peripheral blood and spleen by staining with anti-CD8 (Invitrogen) antibody and SIINFEKL-specific MHCtetramer (H-2 Kb/SIINFEKL 257-264; Beckman Coulter, Brea, CA, USA). Flow cytometric analysis was performed on a FACSCalibur analytical flow cytometer (BD Biosciences) using FlowJo software (Tree Star, Ashland, OR, USA).

\section{Conflict of interest}

U Sahin (founder, chief executive officer) and Christoph Huber (founder) are associated with BioNTech (Mainz, Germany), a company, which develops RNA-based cancer vaccines. U Sahin, A Kuhn, J Kowalska, J Jemielity and E Darzynkiewicz are inventors on a patent application, in which parts of this paper are covered. All other authors declare no potential conflict of interest.

\section{Acknowledgements}

This work was supported by the Combined Project Grant SFB 432 (CH, ÖT, and US), by the GO-Bio funding of the Federal Ministry of Education and Research (BMBF) (US) and by the National Science Support Project 2008-2010 No. PBZ-MNiSW-07/I/2007 (JK, JJ and ED). E Darzynkiewicz is a Howard Hughes Medical Institute International Scholar (Grant No. 55005604). We would like to thank D Ewald and J Kolb for excellent technical assistance.

\section{References}

1 Weide B, Garbe C, Rammensee HG, Pascolo S. Plasmid DNAand messenger RNA-based anti-cancer vaccination. Immunol Lett 2008; 115: 33-42.

2 Yamamoto A, Kormann M, Rosenecker J, Rudolph C. Current prospects for mRNA gene delivery. Eur J Pharm Biopharm 2009; 71: 484-489.

3 Pascolo S. Vaccination with messenger RNA (mRNA). Handb Exp Pharmacol 2008; 183: 221-235.

4 Banchereau J, Steinman RM. Dendritic cells and the control of immunity. Nature 1998; 392: 245-252. 
5 Gilboa E. DC-based cancer vaccines. I Clin Invest 2007; 117: 1195-1203.

6 Kyte JA, Gaudernack G. Immuno-gene therapy of cancer with tumour-mRNA transfected dendritic cells. Cancer Immunol Immunother 2006; 55: 1432-1442.

7 Probst J, Weide B, Scheel B, Pichler BJ, Hoerr I, Rammensee HG et al. Spontaneous cellular uptake of exogenous messenger RNA in vivo is nucleic acid-specific, saturable and ion dependent. Gene Ther 2007; 14: 1175-1180.

8 Weide B, Carralot JP, Reese A, Scheel B, Eigentler TK, Hoerr I et al. Results of the first phase I/II clinical vaccination trial with direct injection of mRNA. J Immunother 2008; 31: 180-188.

9 Weide B, Pascolo S, Scheel B, Derhovanessian E, Pflugfelder A, Eigentler TK et al. Direct injection of protamine-protected mRNA: results of a phase $1 / 2$ vaccination trial in metastatic melanoma patients. J Immunother 2009; 32: 498-507.

$10 \mathrm{Su}$ Z, Dannull J, Yang BK, Dahm P, Coleman D, Yancey D et al. Telomerase mRNA-transfected dendritic cells stimulate antigenspecific CD8+ and CD4+ $\mathrm{T}$ cell responses in patients with metastatic prostate cancer. J Immunol 2005; 174: 3798-3807.

11 Nair SK, Morse M, Boczkowski D, Cumming RI, Vasovic L, Gilboa E et al. Induction of tumor-specific cytotoxic T lymphocytes in cancer patients by autologous tumor RNA-transfected dendritic cells. Ann Surg 2002; 235: 540-549.

12 Princiotta MF, Finzi D, Qian SB, Gibbs J, Schuchmann S, Buttgereit $\mathrm{F}$ et al. Quantitating protein synthesis, degradation, and endogenous antigen processing. Immunity 2003; 18: 343-354.

13 Holtkamp S, Kreiter S, Selmi A, Simon P, Koslowski M, Huber C et al. Modification of antigen-encoding RNA increases stability, translational efficacy, and T-cell stimulatory capacity of dendritic cells. Blood 2006; 108: 4009-4017.

14 Kreiter S, Selmi A, Diken M, Sebastian M, Osterloh P, Schild H et al. Increased antigen presentation efficiency by coupling antigens to MHC class I trafficking signals. J Immunol 2008; 180: 309-318.

15 Cougot N, van Dijk E, Babajko S, Seraphin B. Cap-tabolism'. Trends Biochem Sci 2004; 29: 436-444.

16 Sonenberg N, Hinnebusch AG. Regulation of translation initiation in eukaryotes: mechanisms and biological targets. Cell 2009; 136: 731-745.

17 Pasquinelli AE, Dahlberg JE, Lund E. Reverse $5^{\prime}$ caps in RNAs made in vitro by phage RNA polymerases. RNA 1995; 1: 957-967.

18 Grudzien E, Stepinski J, Jankowska-Anyszka M, Stolarski R, Darzynkiewicz E, Rhoads RE. Novel cap analogs for in vitro synthesis of mRNAs with high translational efficiency. RNA 2004; 10: 1479-1487.

19 Jemielity J, Fowler T, Zuberek J, Stepinski J, Lewdorowicz M, Niedzwiecka A et al. Novel "anti-reverse" cap analogs with superior translational properties. RNA 2003; 9: 1108-1122.

20 Stepinski J, Waddell C, Stolarski R, Darzynkiewicz E, Rhoads RE. Synthesis and properties of mRNAs containing the novel 'anti-reverse' cap analogs 7-methyl(3'-O-methyl)GpppG and 7-methyl (3'-deoxy)GpppG. RNA 2001; 7: 1486-1495.

21 Grudzien-Nogalska E, Jemielity J, Kowalska J, Darzynkiewicz E, Rhoads RE. Phosphorothioate cap analogs stabilize mRNA and increase translational efficiency in mammalian cells. RNA 2007; 13: 1745-1755.

22 Kowalska J, Lewdorowicz M, Zuberek J, Grudzien-Nogalska E, Bojarska E, Stepinski J et al. Synthesis and characterization of mRNA cap analogs containing phosphorothioate substitutions that bind tightly to eIF4E and are resistant to the decapping pyrophosphatase DcpS. RNA 2008; 14: 1119-1131.

23 Mockey M, Gonçalves C, Dupuy FP, Lemoine FM, Pichon C, Midoux P. mRNA transfection of dendritic cells: synergistic effect of ARCA mRNA capping with Poly(A) chains in cis and in trans for a high protein expression level. Biochem Biophys Res Commun 2006; 340: 1062-1068.

24 Rabinovich PM, Komarovskaya ME, Ye ZJ, Imai C, Campana D, Bahceci $\mathrm{E}$ et al. Synthetic messenger RNA as a tool for gene therapy. Hum Gene Ther 2006; 17: 1027-1035.

25 Grudzien E, Kalek M, Jemielity J, Darzynkiewicz E, Rhoads RE. Differential inhibition of mRNA degradation pathways by novel cap analogs. J Biol Chem 2006; 281: 1857-1867.

26 Tcherepanova IY, Adams MD, Feng X, Hinohara A, Horvatinovich J, Calderhead D et al. Ectopic expression of a truncated CD40L protein from synthetic post-transcriptionally capped RNA in dendritic cells induces high levels of IL-12 secretion. BMC Mol Biol 2008; 9: 90.

27 Türeci O, Bian $\mathrm{H}$, Nestle FO, Raddrizzani L, Rosinski JA, Tassis A et al. Cascades of transcriptional induction during dendritic cell maturation revealed by genome-wide expression analysis. FASEB J 2003; 17: 836-847.

28 Yewdell JW, Anton LC, Bennink JR. Defective ribosomal products (DRiPs): a major source of antigenic peptides for MHC class I molecules? J Immunol 1996; 157: 1823-1826.

29 Michiels A, Tuyaerts S, Bonehill A, Corthals J, Breckpot K, Heirman $\mathrm{C}$ et al. Electroporation of immature and mature dendritic cells: implications for dendritic cell-based vaccines. Gene Ther 2005; 12: 772-782.

30 Kreiter S, Konrad T, Sester M, Huber C, Türeci O, Sahin U. Simultaneous ex vivo quantification of antigen-specific CD4+ and CD8+ T cell responses using in vitro transcribed RNA. Cancer Immunol Immunother 2007; 56: 1577-1587.

31 R Development Core Team. R: A Language and Environment for Statistical Computing. R Foundation for Statistical Computing: Vienna, Austria, 2006.

32 Koslowski M, Sahin U, Mitnacht-Kraus R, Seitz G, Huber C, Türeci O. A placenta-specific gene ectopically activated in many human cancers is essentially involved in malignant cell processes. Cancer Res 2007; 67: 9528-9534.

33 Koslowski M, Sahin U, Dhaene K, Huber C, Türeci O. MS4A12 is a colon-selective store-operated calcium channel promoting malignant cell processes. Cancer Res 2008; 68: 3458-3466. 